NSM 01427

\title{
Chronic drug infusion into the scala tympani of the guinea pig cochlea
}

\author{
Gerben G. Kingma *, Josef M. Miller and Michael W. Myers \\ Kresge Hearing Research Institute, University of Michigan, Ann Arbor, MI 48109-0506 (USA) \\ (Received 18 December 1991) \\ (Accepted 23 July 1992)
}

Key words: Cochlea; Chronic drug treatment; Micro-injection system; (Guinea pig)

This research describes a unique, effective and inexpensive delivery system to provide discrete quantities of drugs on a chronic basis to the inner ear. The amount of the drug administered and specific timing of each administration are under investigator control. A micro-injection system mounted atop an animal's head is shown to permit repeated application of agents which effectively block neural responsiveness (tetrodotoxin) on a daily basis for periods up to 2 weeks. Cannulation of the inner ear and chronic delivery of control substances (artificial perilymph) do not affect function. This system may be used to administer drugs to other compartments of the body (e.g., the brain) on a chronic basis for neurophysiologic and neuropharmacologic investigations.

\section{Introduction}

Studies of the effects of chronic local drug application to the cochlea have been hindered by the relative inaccessibility of the temporal bone. In acutely prepared animals, investigators have successfully studied the physiological and histological effects of drugs applied locally on the cochlea and from these studies provided insights into local mechanisms and membrane characteristics of inner ear tissue. One technical approach was the perfusion of agents in a solution of artificial perilymph through the scalae (Nuttall et al., 1977; Mori et al., 1986; Ryan et al., 1987). This method guarantees reasonably even distributiom

Correspondence to: Josef M. Miller, Ph.D., Kresge Hearing Research Institute, University of Michigan, 1301 E. Ann Street, Ann Arbor, MI 48109-0506, USA. Tel.: (1-313) 7648111; FAX: (1-313) 764-0014.

* Current address: Spuistraat 297, 1012 VS Amsterdam, The Netherlands. of the drug through the cochlea and relatively fast elimination of the drug. In other experiments, drugs have been introduced into the cochlea via the round window (Lundman et al., 1987; Morizono et al., 1987; Schachern et al., 1987; Sillman, et al., 1989; Ohlsén et al., 1991). For chemicals with low molecular weights (less than $400 \mathrm{Da}$ ) an agent applied on the round window quickly diffuses into the scala tympani and, in time, will reach the apex and modiolus of the cochlea. With this method, the time and concentration of drug distribution along the cochlea may be variable; however, no direct anatomical changes are induced into the scalae. In addition, injection of a solution directly into the scala tympani through the round window and cannulation of the round window has been used reliably. If only small volumes are infused, only minor changes are made in the perilymph composition and doses may be accurately controlled (Kaplan et al., 1983; Born et al., 1988).

For many experimental questions multiple and long-term drug applications are required. For ex- 
ample, in studies of tetrodotoxin (TTX)-induced suppression of auditory nerve action potentials, the peripheral and central effects of prolonged deprivation for days or weeks would be of significant interest. Techniques developed for acute drug applications are unsuitable for long-term drug applications due to the requirement of repeated anesthesia and potential infection that accompany continued or re-exposure of the cochlea. To avoid some of these complications and accomplish a long-term drug effect, Pasic and Rubel (1989) used Elvax disks that were impregnated with TTX. These disks, positioned on the round window, slowly released TTX on the surface of the membrane allowing diffusion into the perilymph. This technique was shown to release the TTX continuously for a 2-day period. Changes in size and TTX concentration of the disks may lengthen the time over which the agent is released; but based upon our own observations, its ability to cause auditory nerve blockade appears limited to a maximum of 4-5 days.

Cannulae chronically implanted into the tympanic bulla have been used in cats for chronic local drug administration to the surface of the inner ear and, hence, hopefully into the inner ear. Igarashi and Suzuki (1985) injected chlorhexidine into the middle ear three times over a 6-day period. Diffusion from the middle ear into the cochlea led to a chronic application of the agent to the inner ear. However, this technique does not yield precise control over the amount of the agent introduced into the inner ear.

For many studies of local drug effects an implantable osmotic pump (e.g., ALZET, miniseries, ALZA Corp, Palo Alto, CA) with a small cannula can provide a means to introduce small, controlled quantities of an agent into the inner ear (personal observation) or other sites of the body (e.g., Friedman and Shatz, 1990). These devices have been in use for some years (Theeuwes and Yum, 1976), and have the advantages of simplicity, total implantability, reliability, and commercial availability. The disadvantage of these devices is that they do not permit irregular intervals or doses of drug administration, nor the ability to stop and restart drug administration under experimenter control. For many studies the ability to assess recovery, permanent drug effect, and to repeatedly examine the effects of an agent or different quantities of an agent, are necessary.

In the present article, we describe and discuss an infusion system that is capable of infusing multiple dosages of a drug in the guinea pig cochlea over a longer period of time. This system allows multiple infusions directly into the scala tympani of very small amounts of solutions, the volume of which can be varied from injection to injection; and the time of administration is under control of the investigator.
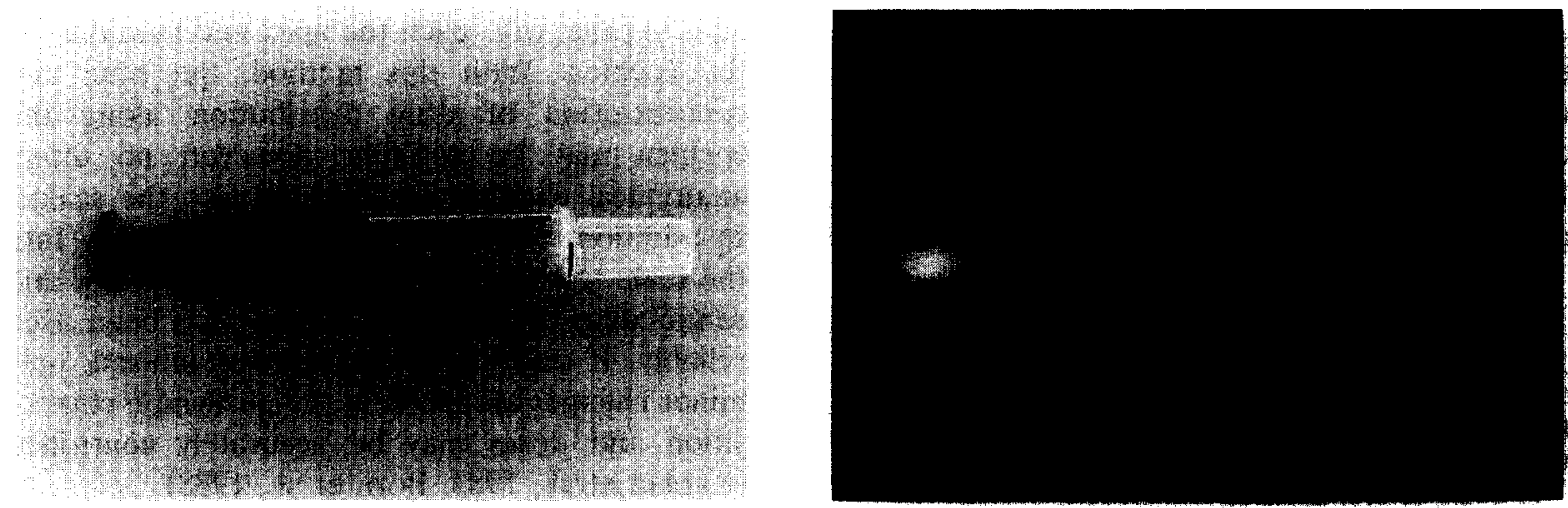

Fig. 1. A: syringe tip and plunger with screw placement for construction of the micro-infusion system body. B: micro-infusion system (MIS) with cannula attached to infusion reservoir 


\section{Materials and methods}

A micro-infusion system (MIS) was designed for implantation into the guinea pig cochlea. We used Hartley strain guinea pigs between 250 and $500 \mathrm{~g}$. The MIS consists of 2 major parts: the micro-injector with screw mechanism for regulation of volume of solution infused, which was anchored to the skull; and the catheter system with infusion tip, which was implanted into the scala tympani.

\section{Materials and fabrication}

The body of the micro-injector was made from the end of a $1-\mathrm{ml} 26 \mathrm{G} 3 / 8 \mathrm{in}$. tuberculin syringe (Becton Dickinson and Company, Rutherford). The plunger was removed and the first $0.5 \mathrm{~cm}$ of the needle holder tip and 'back' $5.8 \mathrm{~cm}$ of the syringe body (from the $0.25 \mathrm{ml}$ mark) were cut off and discarded. This left a section of the syringe body that was $1.5 \mathrm{~cm}$ in length from just behind the tip. This functioned as the body of the microinjector (see Fig. 1A). The rubber tip of the plunger was saved. A no. 4-40 $\times 1 / 2$ in. stainless steel screw with hex nut (Small Parts, Inc., Miami, FL) was then screwed into the hole of the rubber plunger and locked in place with cyanoacrylate adhesive. The rubber plunger, with the screw and nut, was inserted into the syringe until the nut seated against the end of the syringe (Fig. 1B). The nut was fixed to the syringe with autoclavable plastic tape. Turning the screw depressed the plunger and, hence, injected small amounts of the solution.

In order to accommodate the catheter, the diameter of the syringe tip was enlarged sufficiently using a small pin to permit introduction and partial insertion (about $3 \mathrm{~mm}$ ) of a $6-\mathrm{cm}$ length of ( 0.033 outer diameter, 0.014 inner diameter) micro-renathane tubing (Braintree Scientific, Inc.). This catheter was fixed into the syringe tip with cyanoacrylate adhesive, taking care not to plug the catheter with glue.

The infusion tip of the catheter was made from a $1.5-\mathrm{cm}$ length of teflon coating insulation gently stripped from 5T Pt-Ir wire (A-M Systems, Inc., Everett, WA). This teflon tube was then carefully inserted in the distal end of the catheter tube. A drop of liquid silicone rubber (Dow Corning silastic elastomer and Dow Corning catalyst MDX 4-4210) was used to fix the teflon tube to the micro-renathane tubing. In addition, a tiny drop of silicone rubber was applied $0.5 \mathrm{~mm}$ from the end of the infusion tip. This silicone drop limited the extent to which the infusion tip could be introduced into the scala tympani. In addition, it helped seal the fistula created to introduce the cannula and provided a safe point to grasp the system during the implantation procedure. To enhance the hardening process of the silicon rubber, the catheter was baked in an oven at $70^{\circ} \mathrm{C}$ for $20 \mathrm{~min}$. The cathether was then cleaned, checked for leakages by perfusing it with alcohol and distilled water, and autoclaved. Construction of the catheter and evaluation of the end product was performed with the aid of an operating microscope.

\section{Implantation procedure}

Before implantation of the MIS, the injector was filled with the drug solution. This was accomplished by first filling the body of the syringe with the drug solution, taking care to eliminate any trapped air bubbles. The syringe tip with the affixed catheter was then snapped onto the syringe body. The injection screw was turned clockwise until the solution appeared at the infusion tip. Then the tip of the catheter was placed in the solution and extra fluid was drawn into the injector by turning the screw counterclockwise to fill the system to capacity. The total volume of the solution the MIS holds is approximately $0.125 \mathrm{ml}$.

The implantation procedure was performed using aseptic surgical techniques and conformed to the Guidelines of Humane Animal Care. Surgical anesthesia was accomplished using ketamine (40 mg/kg, i.m.) xylazine (10 mg/kg, i.m.) and local infusion of $1 \%$ lidocaine. The animals were given prophylactic antibiotic injections prior to and following surgery (chloromycetin, $10 \mathrm{mg} / \mathrm{kg}$, i.m.). The animal's skull was exposed via a midline incision and the periosteum was removed. Three no. $1-72 \times 5 / 6$ in. stainless steel screws (Small Parts, Inc., Miami, FL) were self-tapped into holes drilled around the bregma. These allowed secure anchoring of the MIS to the skull 
with methyl methacrylate. In addition, an inverted no. $4-40 \times 1 / 2$ in. stainless steel screw was affixed to the skull as a restraining bolt. A postauricular approach was used to expose the cochlea. The round window and basal turn of the cochlea were visualized and a small hole $(0.1 \mathrm{~mm}$ in diameter) was drilled into the basal turn of the cochlea $2 \mathrm{~mm}$ anterior to the round window, allowing access to the scala tympani.

With fine forceps holding the silicon drop, the infusion catheter was inserted into the hole in the lateral wall until the silicon drop was seated against the otic capsule. A small piece of muscle fascia was placed around the scala tympani defect and methyl methacrylate was used to fix the catheter in position at the bulla wall bony defect. The catheter and injector were then secured to the skull with additional methyl methacrylate. The bony defect in the bulla was covered with a small piece of tissue and the wound was closed with interrupted silk sutures. It was found helpful to fix the injector with the injection screw directed caudally, next to the restraining bolt.

The MIS was tested in vitro and in vivo experiments. For in vivo testing a group of 29 guinea pigs was chronically implanted with the MIS into the scala tympani. Eight of these animals received daily injections of $0.5 \mu \mathrm{l}$ of saline, while the rest received daily $0.5-\mu \mathrm{l}$ injections of $25 \mu \mathrm{M}$ tetrodotoxin* in saline (TTX, a reversible sodium-channel blocker). These subjects received daily repeat injections for 8-14 days. In vitro tests of the injector system evaluated the shrinkage of the MIS with sterilization, reliability of minimal dosing, and passive diffusion of catheter contents. The effectiveness of drug treatment using the MIS was evaluated on the basis of evoked response in the auditory system, using the auditory brain-stem response $(\mathrm{ABR})$ and middle latency response (MLR) as the criteria for evaluation. For these studies, evoked responses were recorded from an active epidural recording electrode placed over the vertex, and an indifferent

\footnotetext{
* This dose was selected based upon acute studies as reliably and reversibly providing a more than $70-\mathrm{dB}$ threshold shift in electrophysiological sensitivity in the cochlea.
}

needle electrode over the ipsilateral postauricular area $(A B R)$ or from an active electrode over the contralateral auditory cortex, with the indifferent needle electrode $2 \mathrm{~mm}$ anterior to the bregma (MLR). Stimuli were clicks elicited by a $0.2-\mathrm{ms}$ voltage pulse imposed across an Etymotic Research speaker (ER-3A 10 $\Omega$ ) which was coupled to the external auditory meatus. Stimuli were delivered at $20 / \mathrm{s}$ (ABR) or 5/s (MLR). Physiological responses were amplified $10,000 \times($ Grass P15 and in-house amplifier), filtered $(3 \mathrm{~Hz}-3 \mathrm{kHz}$ bandwidth) and averaged (512, ABR; 256, MLR) using an IBM AT-based signal processor. ABR and MLR input-output ( $\mathrm{I} / \mathrm{O})$ functions were recorded before and after each drug administration.

At termination of the physiological study, 0.5 $\mathrm{ml}$ of methyl blue dye was injected into the cochlea in 2 animals. One and a half days following injection, these and all other animals completing this study were sacrificed and systemically perfused (4\% paraformaldehyde). The cochleas were decalcified ( $2 \%$ EDTA), embedded in Epon, sectioned mid-modiolarly, stained (paragon) and examined under light microscopy. The extent of methyl dye distribution, trauma due to surgery, and the presence of infection were evaluated in these tissues.

\section{Results}

In vitro studies

The micro-injector was found to be easily autoclavable. No major changes in the structure of the injector were observed after the autoclaving. A $20 \%$ shrinkage in length of the cannula was observed. Strength or functionality of the system was not obviously changed by the shrinkage of the tubing. Pre-autoclaving the tubing or the use of silicone tubing would eliminate the problem of the heat-induced shortening.

Using a small screwdriver, reliable infusion of fixed quantities of solution was given. A minimum injection was accomplished by turning the screw approximately $15^{\circ}$. This was the smallest adjustment possible without the screw returning to its original position, due to hysteresis in the 
system created by the friction of the elastic rubber plunger against the sides of the injector. Twenty-four infusions could be delivered with this minimum advance with one turn of the screw. Each injection was 0.3-0.5 $\mu \mathrm{l}$. One full injector reliably yielded approximately 300 infusions.

Although the inner diameter of the infusion tip is very small ( 0.005 in.), fluids of even moderate viscosity easily passed through the tip and no fluid was visible in the tip following infusion. Testing performed with the catheter infusion tip submerged in isotonic solutions demonstrated little or no diffusion in or out of the infusion tip.

\section{In vivo studies}

The MIS was easily implanted into the cochlea and secured to the animal's skull. In some animals, a small amount of intrascalar bleeding was visible after drilling the hole in the lateral wall of the basal turn. This bleeding quickly resolved, possibly by constriction of the cochlear vessels (Hallen et al., 1974). A brief, small outward deflection of the round window was consistently observed upon injection following implantation, reflecting the intracochlear volume and pressure changes. After infusion, the round window returned to its original position without apparent damage (Dohi et al., 1988) and with no observable effect on cochlear electrophysiological function. Examination of sections of the cochlea taken 1.5 days following infusions of methyl blue dye $(0.5 \mathrm{ml})$ into the scala tympani indicated that the dye spread through the majority of the basal turn. While the dye was infused into the scala tympani, traces of the dye were also visible in the scala vestibuli and the scala media of the cochlea.

Fig. 2 illustrates the acoustically evoked (I/O) function of the ABR before and following $1-\mu 1$ injection of saline. The response functions remained stable across repeated injections. We also observed stable acoustic ABR and MLR I/O response functions following implantation and throughout daily injections of $1 \mathrm{ml}$ of saline for more than 1 week. Fig. 3 is a representative example of the MLR I/O functions observed prior to and following administration of TTX. The TTX infusions reliably decreased evoked responsiveness of the system to near zero on a daily

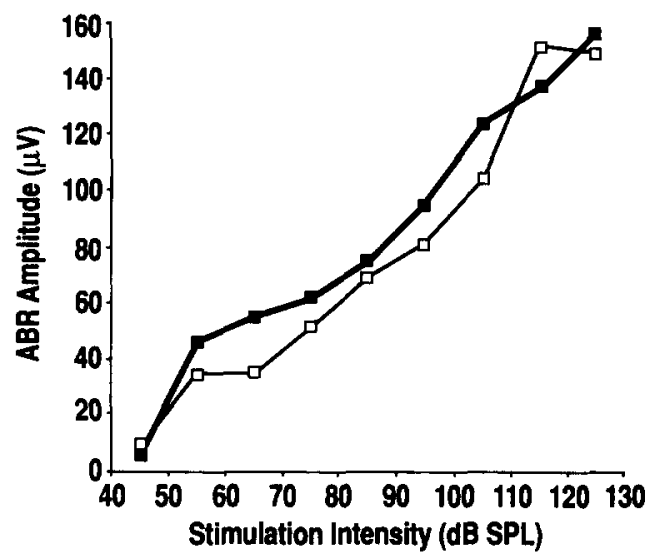

Fig. 2. Click-evoked ABR, amplitude input/output (I/O) functions from 1 representative subject, recorded prior to and following injection of $1 \mathrm{ml}$ of saline into the scala tympani using the micro-injector system (MIS).

basis for approximately $3-5 \mathrm{~h}$ each time the drug was infused into the scala in all subjects. After injection complete recovery was observed; during this recovery, in spite of the presence of the catheter, no evidence for passive diffusion of the drug was observed. The MIS worked reliably for a period of 6-14 days after the implantation in the 29 animals in which it was tested. In 24 of the treated animals, the $\mathrm{I} / \mathrm{O}$ function observed fol-

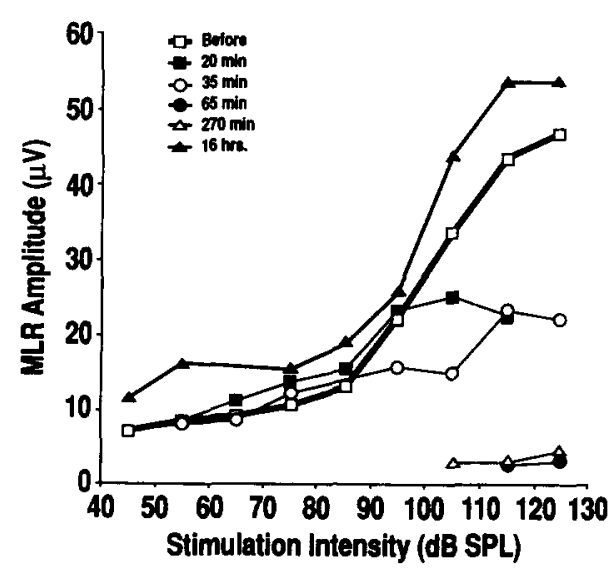

Fig. 3. Click-evoked MLR, input/output (I/O) functions from 1 representative subject recorded before and following a single $0.5-\mu \mathrm{l}$ injection of $25 \mu \mathrm{M}$ tetrodotoxin into the scala tympani via the micro-infusion system (MIS). Recordings were taken at 20, 35, 65 and $270 \mathrm{~min}$ and $16 \mathrm{~h}$ following drug injection. 
lowing termination of the series of daily injections was identical to the function observed prior to implantation and treatment. Terminal $\mathrm{I} / \mathrm{O}$ functions were not obtained for 5 subjects; these did not survive the experimental paradigm, and 2 (including one saline subject) showed signs of middle ear infection.

The 2 animals first implanted with the MIS showed clear drug effects, which ceased after 6 and 8 days. These subjects were re-implanted with a new MIS with no decrease in auditory function from the surgery. The new MIS allowed reliable drug infusion with clear effects on cochlear function.

The 24 implanted animals that completed the study were sacrificed after the infusion period and prepared for histological study. Of the 24 implanted ears, 2 showed clear signs of labyrinthitis. The ears of the remaining subjects showed no signs of infection. In most of the cochleas, a thin layer of fibrous tissue was formed where the infusion tip entered the lateral wall. This fibrous tissue growth appeared to serve as a seal, closing the fistula left between the infusion catheter and the defect in the lateral wall. In no subject was damage to the basilar membrane observed in the vicinity of the catheter tip; and vascular tissues of the lateral wall immediately adjacent to the defect were normal in appearance.

\section{Discussion}

The MIS may provide a strategy to assess the effect on cochlear function, of agents which require chronic study. This approach permits study of agents that require repeated chronic application, long-term recovery functions, reproducibility of an effect in the same preparation and, with re-implantation, study of multiple agents in the same preparation.

In contrast to the Elvax disk system (Pasic and Rubel, 1989) and the mini-osmotic pump (Friedman and Shatz, 1990), the MIS does not yield a constant low-level drug diffusion into the perilymph, but the drug solution is infused by singleinfusion doses. Passive diffusion of the drug from the catheter to the perilymph between infusions are apparently minor, as observed in the in vivo studies. According to the characteristics of the drug, and the frequency and concentration of infusions, drug levels in the perilymph will fluctuate significantly over time. This feature makes the system useful for experiments in which drug effects are required for a restricted amount of time per day, but in which normal physiological conditions are required between drug doses. Moreover, this system permits a variable volume of drug to be injected at each treatment. In our experiments (Kingma et al., in preparation), we used TTX to depress acoustic and electrically evoked action potentials. We were able to depress the system significantly for a 3 -h period after a single infusion. After this period, action potentials returned to normal and the system was excitable by normal physiological stimuli. For a more continuous drug effect with such a short-lasting agent, infusions may be repeated more frequently, i.e., every $2-3$ h.

Mechanical damage to the cochlea was not a major factor associated with this technique. The hole in the lateral wall for the infusion tip is positioned $2 \mathrm{~mm}$ anterior to the round window, well below the basilar membrane. When inserted into the defect, the infusion tip position is restricted by the silastic guard, in this study $0.5 \mathrm{~mm}$ beyond the tip; however, this distance may be varied. One half of a millimeter consistently placed the tip in the middle of the scala tympani with no direct mechanical trauma to structures inside the scala. Damage to lateral wall collecting venules demonstrated no immediate influence on the physiological measures we applied.

The micro-injector has been designed as a closed system that does not allow for refilling after implantation. This is an advantage in that sterility of the solution is assured. Moreover, if the system is refillable, pressure changes inside the system would be difficult to avoid during refilling. In addition to diffusion of drug during refilling, these pressure changes could also damage cochlear structures. On the other hand, the disadvantage of this design is that effective drug life in solution is a limiting factor for some studies and it is difficult to test multiple agents in the same preparation. Moreover, part of the system is 
implanted under the animals skin and the other is embedded in dental acrylic that is in direct contact with the skull. Thus, most of the drug solution is kept in solution at or near body temperature. Some drugs, when kept in solution will degrade quickly at these temperatures. As demonstrated, however, re-implantation of the MIS can be performed to replace the drug under study.

In our experiments saline was used as the carrier. The turnover of the perilymph, in combination with the relatively small amount of saline infused, led to no detectable effect of the vehicle on cochlear function. Sterile artificial perilymph obviously may be a better alternative for future long-term studies. During infusion of the solution, the volume of the perilymph will increase. This volume increase is reduced in a short time, in part because of the patent nature of the cochlear aqueduct in the guinea pig, which permits perilymph to move into the CSF. The effect of flow in the opposite direction when a fistula is made in one of the scala is well documented (Moscovitch et al., 1973; Desjardin-Giasson et al., 1985; Ohyama et al., 1988).

There was no significant change observed in the ABR/MLR I/O functions before and after implantation and after repeated infusion periods. This indicates that a small defect may be made in the lateral wall and drug solutions may be infused on a daily basis, with no major change in the physiological characteristics of the system as measured by these responses.

\section{Acknowledgements}

This work was supported in part by PHS Grant DC00274. The authors would also like to thank Yvonne Beerens and Denise D. Dyson for their assistance in the preparation of this manuscript.

\section{References}

Born, D.E. and Rubel, E.W. (1988) Afferent influences on brain stem auditory nuclei of the chicken: presynaptic action potentials regulate protein synthesis in nucleus magnocellularis neurons. J Neurosci., 8: 901-919.
Desjardins-Giasson, S., Beaubien, A.R. and Cauchy, M.J. (1985) Effect of pentobarbital anesthesia on amikacin concentrations in plasma and perilymph and evaluation of multiple sampling in perilymph of guinea pigs. Antimicrob. Agents Chemother., 27: 720-723.

Dohi, T., Horiuchi, T., Masuzawa, T., Fujiki, T., Futaki, T., Hara, M. and Nomura, Y. (1988) Development of pressure monitoring and controlling system for quantitative analysis of experimentally induced perilymph fistula. Acta Otolaryngol. Suppl. 456: 74-82.

Friedman, S. and Shatz, C.J. (1990) The effects of prenatal intracranial infusion of tetrodotoxin on naturally occurring retinal ganglion cell death and optic nerve ultrastructure. Eur. J. Neurosci., 2: 243-253.

Hallen, O., McPherson, D.L., Axelsson, A. and Miller, J.M. (1974) Long-term morphological and electrophysiological effects of small mechanical lesions in the guinea pig cochlea. Acta Otolaryngol., 78: 309-320.

Igarashi, Y. and Suzuki, J. (1985) Cochlear ototoxicity of chlorhexidine gluconate in cats. Arch. Otorhinolaryngol., 242: $167-176$

Kaplan, M.S., Szaro, B.G. and Weiss, T.F. (1983) Components of cochlear electric responses in the alligator lizard. Hear Res., 12: 323-351.

Kingma, G.G., Myers, M.W., Miller, J.M., Altschuler, R.A. and Hartshorn, D.O. (1991) The effect of tetrodotoxin on electrically induced damage in the cochlea. in preparation.

Lundman, L., Holmquist, L. and Bagger-Sjoback, D. (1987) Round window membrane permeability: an in vitro model. Acta Otolaryngol., 104: 472-480.

Mori, N., Ohta, K. and Matsunaga, T. (1986) The effect of vasopressin upon the cochlear potentials in the guinea pig. Acta Otolaryngol., 101: 217-223.

Morizono, T., Smith, R., Chap, T., Canafax, D. and Giebink, S. (1987) Ototoxicity of topically applied drugs used in otitis media treatment. In: D.J. Lin, C.D. Bluestone, J.O. Klein and J.D. Nelson (Eds.) Recent Advances in Otitis Media, Proc. 4th International Symposium, B.C. Decker, Inc., Philadelphia, PA, pp. 1-4.

Moscovitch, D.H., Gannon, R.P. and Laszlo, C.A. (1973) Perilymph displacement by cerebrospinal fluid in the cochlea. Ann. Otol. Rhinol. Laryngol., 82: 53-61.

Nuttall, A.L., Marques, D.M. and Lawrence, M. (1977) Effects of perilymphatic perfusion with neomycin on the cochlear microphonic potential in the guinea pig. Acta Otolaryngol., 83: 393-400.

Ohlsén, K.A., Baldwin, D.L., Nuttall, A.L. and Miller, J.M. (1991) Influence of topically applied adrenergic agents on cochlear blood flow. Circ. Res., 69: 509-519.

Ohyama, K., Salt, A.N. and Thalmann, R. (1988) Volume flow rate of perilymph in the guinea pig cochlea. Hear Res., 35: 119-129.

Pasic, T.R. and Rubel, E.W. (1989) Rapid changes in cochlear nucleus cell size following blockade of auditory nerve electrical activity in gerbils. J. Comp. Neurol., 283: 474480. 
Ryan, A.F., Schwartz, I.R., Helfert, R.H., Keithley, E. and Wang, Z.X. (1987) Selective retrograde labeling of lateral olivocochlear neurons in the brainstem based on preferential uptake of $\left[{ }^{3} \mathrm{H}\right]-\mathrm{D}$-aspartic acid in the cochlea. J. Comp. Neurol., 255: 606-616.

Schachern, P., Paparella, M., Coycoolea, M., Duvall, A. and Choo, Y.-B. (1987) The permeability of the round window membrane during otitis media. Arch. Otolaryngol. Head Neck Surg., 113: 625-629.
Sillman, J.S., Masta, R.I., LaRouere, M.J., Nuttall, A.L. and Miller, J.M. (1989) Electrically stimulated increases in cochlear blood flow. II. Evidence of neural mediation. Otolaryngol. Head Neck Surg., 101: 362-374.

Theeuwes, F. and Yum, S.I. (1976) Principles of the design and operation of generic osmotic pumps for the delivery of semi-solid or liquid formulations. Ann. Biomed. Eng., 4: 343-353. 